\title{
Large-scale pattern of genetic differentiation within African rainforest trees: insights on the roles of ecological gradients and past climate changes on the evolution of Erythrophleum spp (Fabaceae)
}

\author{
Jerome Duminil ${ }^{1,2^{*}}$, Richard P Brown ${ }^{3}$, Eben-Ezer BK Ewédjè ${ }^{1,4}$, Patrick Mardulyn ${ }^{1}$, Jean-Louis Doucet ${ }^{5}$ \\ and Olivier J Hardy ${ }^{1}$
}

\begin{abstract}
Background: The evolutionary events that have shaped biodiversity patterns in the African rainforests are still poorly documented. Past forest fragmentation and ecological gradients have been advocated as important drivers of genetic differentiation but their respective roles remain unclear. Using nuclear microsatellites (nSSRs) and chloroplast non-coding sequences (pDNA), we characterised the spatial genetic structure of Erythrophleum (Fabaceae) forest trees in West and Central Africa (Guinea Region, GR). This widespread genus displays a wide ecological amplitude and taxonomists recognize two forest tree species, E. ivorense and E. suaveolens, which are difficult to distinguish in the field and often confused.
\end{abstract}

Results: Bayesian-clustering applied on nSSRs of a blind sample of 648 specimens identified three major gene pools showing no or very limited introgression. They present parapatric distributions correlated to rainfall gradients and forest types. One gene pool is restricted to coastal evergreen forests and corresponds to E. ivorense; a second one is found in gallery forests from the dry forest zone of West Africa and North-West Cameroon and corresponds to West-African E. suaveolens; the third gene pool occurs in semi-evergreen forests and corresponds to Central African E. suaveolens. These gene pools have mostly unique pDNA haplotypes but they do not form reciprocally monophyletic clades. Nevertheless, pDNA molecular dating indicates that the divergence between E. ivorense and Central African E. suaveolens predates the Pleistocene. Further Bayesian-clustering applied within each major gene pool identified diffuse genetic discontinuities (minor gene pools displaying substantial introgression) at a latitude between 0 and $2^{\circ} \mathrm{N}$ in Central Africa for both species, and at a longitude between $5^{\circ}$ and $8^{\circ} \mathrm{E}$ for $E$. ivorense. Moreover, we detected evidence of past population declines which are consistent with historical habitat fragmentation induced by Pleistocene climate changes.

Conclusions: Overall, deep genetic differentiation (major gene pools) follows ecological gradients that may be at the origin of speciation, while diffuse differentiation (minor gene pools) are tentatively interpreted as the signature of past forest fragmentation induced by past climate changes.

Keywords: Cluster analysis, Fragmentation, Last glacial maximum, Phylogeography, Range expansion, Refugium, Species delimitation, Tropical rainforest

\footnotetext{
*Correspondence: jduminil@ulb.ac.be

'Service Evolution Biologique et Ecologie, CP160/12, Faculté des Sciences,

Université Libre de Bruxelles, 50 Av. F. Roosevelt, 1050 Brussels, Belgium

${ }^{2}$ Bioversity International, Forest Genetic Resources Programme, Sub-Regional

Office for Central Africa, P.O. Box 2008 Messa, Yaoundé, Cameroon

Full list of author information is available at the end of the article
} 


\section{Background}

The evolution of African rainforest species is still poorly understood. The Guineo-Congolian tropical forests cover a wide area, forming one block extending from Sierra Leone to Ghana in West Africa, and another block from southern Nigeria to the Albertine rift in Central Africa. According to White [1], three main types of Guineo-Congolian rainforests occur along a rainfall gradient displaying a coastal-inland orientation: evergreen forest ("Hygrophilous coastal evergreen rainforest"; rainfall $>2000 \mathrm{~mm}$ ); semi-evergreen forest ("Mixed moist semi-evergreen rainforest"; rainfall between 1600 and $2000 \mathrm{~mm}$ ); and dry forest ("Drier peripheral semievergreen rainforest"; rainfall between 1200 and $1600 \mathrm{~mm}$ ). Furthermore, the Guineo-Congolian phytochoria has been divided into three sub-centres of endemism along a West-east axis based on patterns of species distribution and endemism [2]. These are: i) the Upper Guinea region (UGR) in West Africa, ii) the Lower Guinea region (LGR) to the West of Central Africa, and iii) the Congolian region to the East of Central Africa. The UGR and LGR are separated by a broad savanna corridor, also known as the Dahomey Gap (Benin, Togo, and South East of Ghana; Figure 1). A range of factors, including spatial ecological gradients, historical climate changes, and physical barriers to gene flow might have contributed to the organization of the diversity within the African rainforest, both within and among species. Given the scarcity of palynological data in the lowland tropics, molecular phylogenetics and population genetics approaches can provide major insights into the relative influence of these factors. The present work aims to provide a better understanding of the evolutionary history of the Guinean tropical forests by addressing the phylogeography of two sister-tropical tree species across the UGR and LGR (hereafter referred to together as the Guinea region: GR).

Genetic studies of plastid and nuclear DNA in plant species from the LGR have already demonstrated that within-species genetic diversity often displays spatial discontinuities leading to geographically coherent gene pools or clades, even in the absence of gaps in the distribution of the species [3-8]. However, the distribution of genetic diversity over the entire GR and the origin of this structuring remain largely unknown. Two main hypotheses may explain these spatial genetic discontinuities: (i) the presence of ecological gradients driving adaptive differentiation ('ecological gradient hypothesis'), (ii) forest fragmentation/expansion following climatic oscillations driving differentiation by genetic drift between forest refuges ('forest refuge hypothesis').

The ecological gradient hypothesis posits that environmental gradients can induce parapatric speciation (or ecotypic differentiation) without the need for population isolation. This hypothesis has been supported in a few African animal species (e.g. [9]) and African plants (e.g.

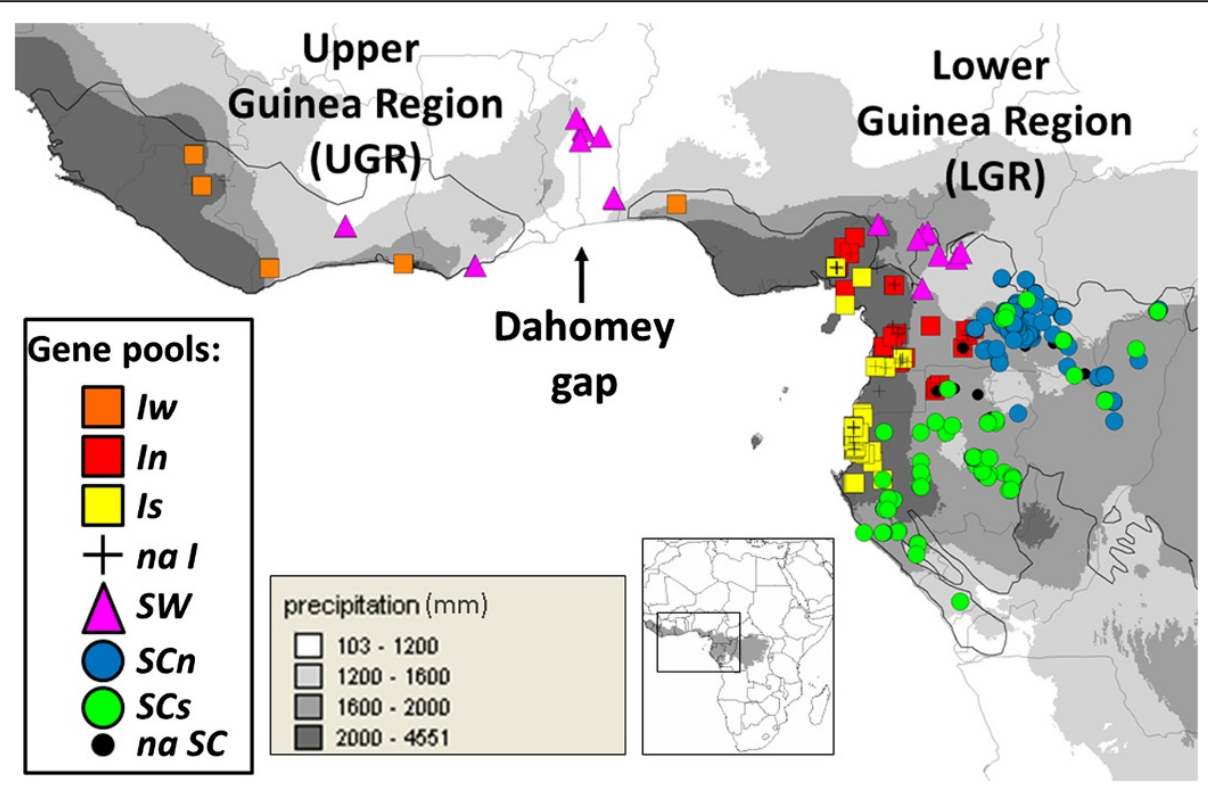

Figure 1 Sampling of the Erythrophleum individuals within the Guinean Region (GR) and assignment to gene pools according to the Bayesian clustering analyses. The levels of rainfall are indicated on the map, providing the approximate distribution of evergreen (rainfall $>2000 \mathrm{~mm}$ ), semi-evergreen (1600-2000 mm) and dry rainforests (1200-1600 mm). The black line is an approximation of the current distribution of wet rainforests. The distribution of major and minor gene pools as inferred from nSSRs is indicated as follow. Purple triangles: SW gene pool, blue and green circles: respectively SCn and SCS gene pools; orange, red and yellow squares: respectively IW, In, Is gene pools; crosses (na I): $E$. ivorense individuals not attributed to any of minor gene pools I; black dots (na SC): E. suaveolens individuals not attributed to any of minor gene pools SC. 
[10]). The forest refuge hypothesis posits that the cyclic fragmentation and re-expansion of lowland forests in response to Pleistocene climatic change has been a main driver for species diversification or population differentiation. The climate was drier in tropical Africa during the last glacial episode (i.e., 110000 to 12000 years BP, with a glacial maximum between 26000 and 19000 years BP) causing an expansion of dry and/or mountain forests and savannas that would have constituted barriers to gene flow among the different rainforest fragments [11,12]. The geographic locations of these refugia are highly debated. Humid conditions had initially returned by $c a .14500$ years $\mathrm{BP}$, reaching a maximum between 9000 and 6000 years $\mathrm{BP}$. This corresponds to the so-called Holocene African humid period which was characterised by a maximal extension of rainforests [13]. At this time, between $c a .8400$ and 4500 years BP, even the Dahomey Gap was covered by the rainforest [14]. A significant increase in aridity at the end of the mid-Holocene led to the retreat of the rainforests and the reopening of the Dahomey Gap [15].

Erythrophleum (Fabaceae-Caesalpinioideae) is a pantropical woody genus with representative species from North-East Asia (E. fordii, E. succirubrum, E. densiflorum), Australia (E. chlorostachys), Madagascar (E. couminga) and Africa (E. ivorense, E. suaveolens, E. africanum). In this study we focus on the two recognized African forest species: Erythrophleum ivorense A. Chev. (syn. E. micranthum Harms) and E. suaveolens (Guill. et Perr.) Brenan (syn. E. guineense G. Don.). E. ivorense occurs in the UGR and the LGR, from Gambia to Gabon, with a distribution discontinuity between the UGR and the LGR, being totally absent from the Dahomey Gap [16]. This species is found in evergreen forests and requires high rainfall $[8,17,18]$. $E$. suaveolens is more widespread, being found from Senegal to Sudan and Kenya and southward to Mozambique and Zimbabwe [16,17]. Hence, it is present in all three subcentres of endemism of the Guineo-Congolian phytogeographic region and also occurs in adjacent peri-forest areas. It occupies a wider range of climates than $E$. ivorense and is found in semi-evergreen forests (in Central Africa; see [8]) and in gallery forests (outside of Central Africa; $[16,17,19]$ ). Contrary to $E$. ivorense, its distribution is continuous from West to Central Africa, occurring also in the Dahomey Gap [16]. The two species are difficult to distinguish in the field in the absence of reproductive organs, and are grouped under the same timber trade name "tali". In Central Africa, the literature contains several inconsistencies concerning their respective distributions and ecological requirements. Nevertheless, Duminil et al. [8] demonstrated that plastid DNA (pDNA) represents an efficient barcode to identify the two species in the LGR where their respective parapatric distributions are correlated with the rainfall gradient. In West Africa, the two species are reported to occur in contrasting habitats $(E$. ivorense in evergreen forests and
E. suaveolens in forest galleries) and are not expected to be found in sympatry [17]. A third related African species, E. africanum (Welw. ex Benth.) Harms, is distributed within the Zambezian and Sudanian regional centres of endemism (sensu White [2]), from Senegal to Sudan, Kenya and Tanzania and southward to the Transvaal region, occurring in woodland savannas but never in forests. Nuclear DNA sequences indicate that African Erythrophleum species form a clade in which E. ivorense and E. suaveolens are more related to each other than to $E$. africanum (J. Duminil, unpublished).

The wide ecological amplitude and geographic distribution of E. ivorense and E. suaveolens within the GR allow analysis of the influence of ecological gradients and past population fragmentation on patterns of genetic differentiation. As taxonomists describe two species which are often confused (at least in Central Africa), we hypothesize that genetic variation might display diffuse discontinuities. Sharp discontinuities (i.e. occurrence of gene pools without introgression) would be expected between isolated reproductive entities, like species following the Biological Species Concept or populations that have been geographically isolated over a long period. Diffuse genetic discontinuities (i.e. gene pools with evidence of substantial introgression) would occur between populations that were (i) recently separated (e.g. by the formation of the Dahomey gap) or (ii) formerly isolated (e.g. in forest refugia) but subsequently came into secondary contact or (iii) parapatric, and adapted to different environments but still maintain some gene flow, in which case they may form a stable hybrid zone.

Here we use nSSRs and pDNA sequences to (i) identify major gene pools (sharp discontinuities) and minor gene pools (diffuse discontinuities) within the GR region and check whether they can be matched to taxonomic species, and (ii) verify whether genetic discontinuities can be explained by current climatic gradients (ecological gradient hypothesis) and/or by Pleistocene climatic changes (forest refuge hypothesis and role of the Dahomey Gap). We attempt to gain complementary insights into the evolution of Erythrophleum (i) by assessing the congruence between gene pools assessed from biparentally-inherited nSSRs and maternally-inherited pDNA haplotypes and clades, (ii) by estimating the divergence times between gene pools using a fossil-calibrated pDNA phylogenetic tree, and (iii) by testing for genetic signature of past demographic changes using nSSRs.

\section{Methods}

\section{Sampling and DNA extraction}

Leaves or cambium were sampled from 648 individuals of E. ivorense and E. suaveolens from the LGR and the UGR (sensu White [2]). As most samples could not be identified taxonomically using morphological traits, 
except samples from Central Africa [8], they were treated as a blind sample for genetic analyses.

Correspondence with taxonomic species is based, in Central Africa, on a previous published work [8]. In West Africa, we considered a priori that samples from the evergreen forest area $(>2000 \mathrm{~mm}$ rainfall, Figure 1$)$ must belong to $E$. ivorense while those from the dry forest area $(<1600 \mathrm{~mm}$ rainfall) belong to $E$. suaveolens, in accordance with the known ecology of the species in West Africa [17]. For each sample, the annual precipitation of the sampling location was recovered from WorldClim (variable Bio12).

Additional E. africanum samples from Benin (N10.21 E1.21 and N10.68 E1.63), E. chlorostachys (F.Muell.) Baill. from Australia (S12.49 E130.99) and E. fordii Oliv. from China (three individuals, one from Fujian-N24.1 E117.4-, one from Guangdong-N23.6 E114.7-, one from Guangxi-N22.1 E108.3-) were used as outgroups in phylogenetic analyses. Total DNA was isolated with the NucleoSpin ${ }^{\circ}$ plant kit (Macherey-Nagel).

\section{Microsatellite genotyping and diversity indices}

Nine microsatellite loci (namely Ery1, Ery3, Ery4, Ery6, Ery7, Ery14, Ery 17, Ery18 and Ery23) were amplified for 648 individuals of $E$. ivorense or E. suaveolens using the protocol described in [20].

As the concept of a population is difficult to apply in tropical rainforests, individuals were arbitrarily grouped into 34 populations according to their geographic coordinates. Observed and expected heterozygosities, inbreeding coefficient $\left(F_{\mathrm{IS}}\right)$ and allelic richness $\left(\mathrm{A}_{\mathrm{O}}\right)$ were estimated for each population using FSTAT [21]. The frequency of null alleles per locus and per population was estimated in the 18 populations represented by at least 10 individuals by maximum likelihood following [22] using the program FREENA [23].

\section{Identifying genetic discontinuities}

We used the Bayesian assignment program STRUCTURE [24] to identify differentiated gene pools without prior spatial information on the origin of samples. We used a two-step approach to identify (i) major gene pools showing no or negligible introgression (sharp boundaries) and (ii) minor gene pools displaying introgression (diffuse boundaries) within each major gene pool. Hence, analyses were first run on the whole set of nSSR genotypes using the no admixture and independent allele frequencies model, to avoid overestimation of $K$ [25] and because gene pools having diverged for long may have independent allele frequencies. The admixture model was also tested and gave similar results (data not shown). There were 20 independent iterations for each imposed number of gene pools $K$ varying from 1 to 10, which were summarized using CLUMPP v.1.1.2 [26]. The total MCMC chain length was 40000 steps with the first 10000 steps being discarded as burn-in [27]. To assess the optimal value of $K$ we considered the log-likelihood of the data according to $K$, but also the number of individuals that were not assigned to a gene pool above a probability threshold of 0.8 . The latter criterion was important to identify well isolated gene pools (few or no admixed individuals).

Further STRUCTURE analyses were carried out within each major gene pool using the same procedure except that we used an admixture model with correlated allele frequencies, in which the fraction of ancestry from each gene pool was estimated for each individual. A uniform prior was used whereby the individual admixture alpha parameter was set to be equal for all gene pools. Here, the optimal $K$ was assessed by the log-likelihood of the data only.

\section{Patterns of genetic differentiation}

Fixation indices $\left(F_{\mathrm{ST}}\right.$ and $\left.R_{\mathrm{ST}}\right)$ between pairs of populations or gene pools were computed using SPAGeDi 1.3 [28]. $F_{\mathrm{ST}}$ is based on allele identity while $R_{\mathrm{ST}}$ is an analogue of $F_{\mathrm{ST}}$ based on allele size. $R_{\mathrm{ST}}$ is expected to be larger than $F_{\mathrm{ST}}$ if stepwise mutations have contributed to differentiation, as in the case of ancient isolation [29]. The occurrence of this pattern was tested using random permutations of allele sizes among allelic states [29].

\section{pDNA genetic diversity distribution}

We characterized the genetic variation at the trnC-petN1R inter-genic fragment, as described in [8], on a sub-set of 297 individuals representative of all defined populations (Additional file 1).

We also sequenced the matK gene and the trn $L$ intron using the matK1R-matK3F and trnLc-trnLd primer pairs (respectively Ki-Joong Kim, unpublished; and [30]). Sequences were obtained for 72 (matK) and 66 individuals (trnL) arbitrarily selected from the different gene pools defined by the Bayesian clustering analyses described above. All three pDNA fragments were also sequenced for $E$. africanum, E. chlorostachys and E. fordii.

\section{Phylogenetic network reconstruction}

A median joining network [31] for the trnC-petN1R inter-genic fragment was constructed using the software NETWORK version 4.5.1.6 (Fluxus Technology Ltd). All polymorphic characters were considered (single nucleotide polymorphism, insertion-deletion and simple sequence repeat) except for a small hypervariable portion of the trnC-petN1R inter-genic fragment that could not be unambiguously aligned. All characters were given equal weight. Samples of $E$. africanum were used to root the network (unambiguous nucleotide sequence alignment was not possible for E. chlorostachys or E. fordii, and these were discarded from the analysis). 


\section{Molecular dating analyses}

The matK gene and the trnL intron were used in the dating analyses because they have already been used by Bruneau et al. [32] to date a phylogenetic tree of Fabaceae species, with calibration points specified from fossil dates. Here we focused on the part of the phylogenetic tree that includes Erythrophleum (Figure four in [32]). We obtained $\operatorname{trn} L$ and matK sequences from GenBank for a set of Fabaceae species closely related to Erythrophleum, and attempted to obtain examples from the most divergent lineages within the Erythrophleum clade (Figure four in [32]). For E. ivorense and E. suaveolens sequences, we used a single sample per major gene pool.

The phylogeny and divergence times were estimated using the Bayesian MCMC software package BEASTv1.6.1 [33]. The HKY (for trnL) and the GTR (for each of the three matK codon positions) models of DNA substitution were used with a gamma distribution for among site rate variation (these were the best supported models under the AIC criterion in jModelTest [34]). We constrained some taxa to be monophyletic following unequivocal results in a previous study [32] (see Figure 2).

Rates were specified from the uncorrelated lognormal relaxed molecular clock and a birth-death model was used to specify the prior on times. Prior information on divergence times was obtained from a previous review of fossil-information (paleobotanical records) [31] and used to specify calibrations on specific nodes. Given the age of the root, and the scale dependence of some components of the model, it was reasonable to define 1 time unit = $10 \mathrm{Ma}$ (rather than $1 \mathrm{Ma}$ ). Priors on four node ages (nodes $\mathrm{O}, \mathrm{P}, \mathrm{R}$ and $\mathrm{S}$; see below for their designation) were specified from the Normal distribution, $\mathrm{N}(4.5,1)$, which was asymmetrically truncated by the

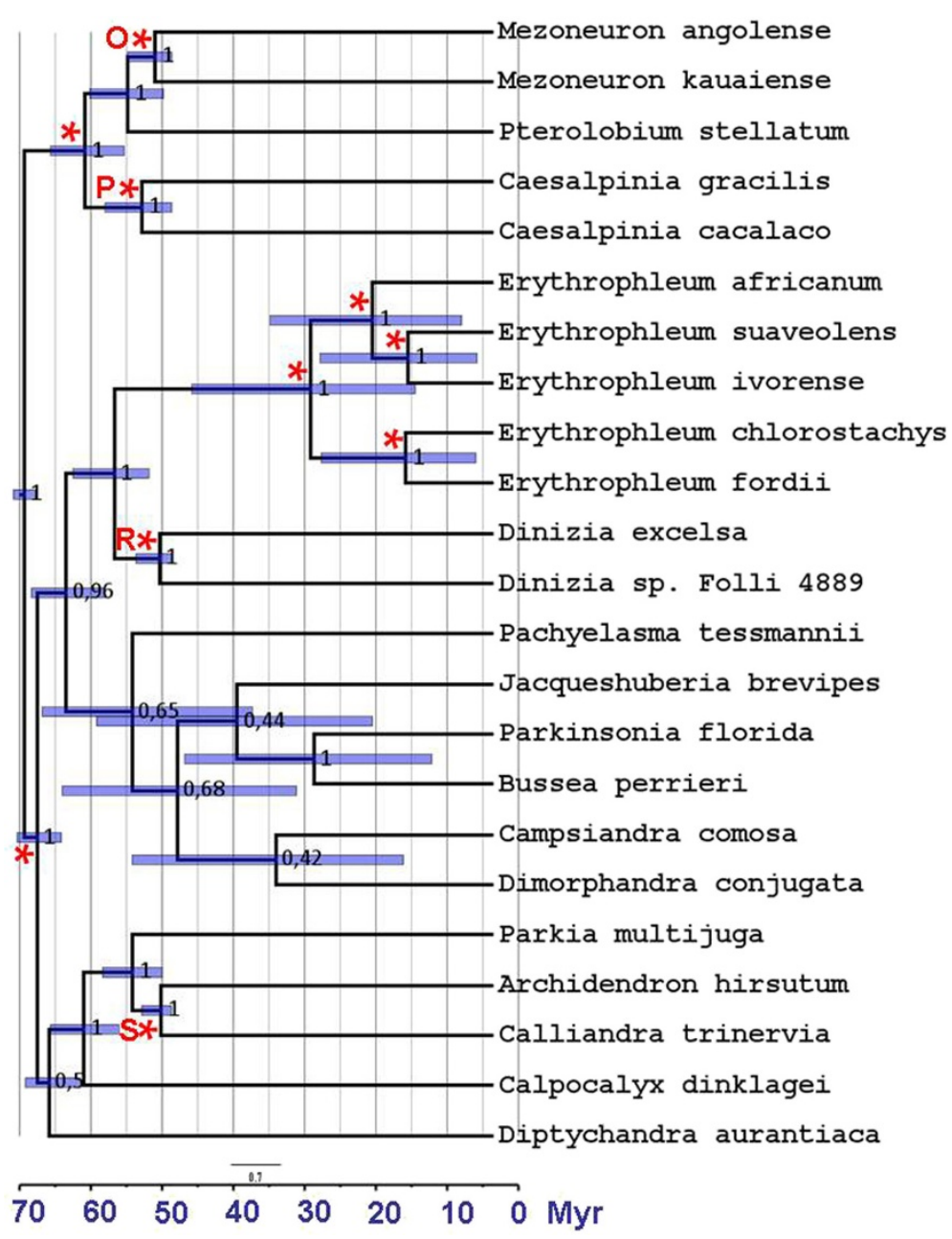

Figure 2 Divergence time chronogram of the Bayesian maximum clade credibility tree based on the chloroplast fragments matK and trnL. The blue bars represent the $95 \%$ highest posterior density intervals for each divergence time. Fossil calibration nodes are indicated by letters (P, O, R and S) as in [32]. Nodes constrained to be monophyletic are indicated by *. Numbers at internal nodes represent clades posterior probabilities. 
uniform distribution, $\mathrm{U}(4.5,5.6)$. The minimum value of this constraint was justified because there is fossil evidence that divergence within all of these clades began $>45 \mathrm{Ma}$, reviewed in [31]. Also, the estimated divergence times at more basal nodes than $\mathrm{O}, \mathrm{P}, \mathrm{R}$ and $\mathrm{S}$ are estimated to be around $56 \mathrm{Ma}$. Specifically, earliest divergence time within the Caesalpinia clade is $55.9 \mathrm{Ma}$ [31], while the earliest divergence time for nodes $\mathrm{R}$ and $\mathrm{S}$ must be younger than the most recent common ancestor (MRCA) of the Dimomorpha, Mimosoideae, Tachigali, and Peltophorum clades which has been estimated at $56.3 \mathrm{Ma}$ [31]. To summarize, our analysis constrained the ages of the following four nodes to be between 45 and $56 \mathrm{Ma}$, with greatest prior density around $45 \mathrm{Ma}$ and lowest density around $56 \mathrm{Ma}$ : (1) the most recent common ancestor (MRCA) of the Caesalpinia clade (node P in [31]), (2) the MRCA of the Mezoneuron clade (node O), (3) the MRCA of the Dinizia clade (node $\mathrm{R})$, (4) the MRCA of the Ingeae clade (node S). In addition, we followed [31] by constraining the root node (equivalent to the most basal divergence within the legumes) to be close to $65 \mathrm{Ma}$, using a gamma distribution. We examined the effects of three different Gamma priors placed on the root: G $(65,0.1), G(650,0.01)$ and $G(6500,0.001)$. The first of these represents the loosest calibration and the last represents the tightest: $95 \%$ of the G $(65,0.1)$ distributions lies within the range $52-79 \mathrm{Ma}$, while $95 \%$ of the G (6500, 0.001 ) lies within $63-66 \mathrm{Ma}$.

Two MCMC analyses were run each for 100 million generations sampled at 5000 step intervals. We used Tracer v1.4 [33] to assess convergence, estimate effective sample sizes (ESS), and examine the posteriors of all parameters. The first 500 samples were discarded as burn-in.

\section{Detection of past variation in population size}

Microsatellite markers were used to detect signals of demographic decline or expansion using the Bayesian inferential method of Storz and Beaumont [35] implemented in MSVAR1.3. The method relies on the full allelic distribution and takes into account relative sizes of microsatellite alleles, with mutation rates and demographic parameters free to vary among loci. Allelic frequencies are used to estimate the current effective population size $\left(\mathrm{N}_{0}\right)$, the ancestral effective population size $\left(\mathrm{N}_{1}\right)$, the number of generations since the demographic change $(\mathrm{Ta})$ and the mutation rate $(\mu)$. In separate analyses, both $a$ priori defined populations (Additional files 2 and 3) and gene pools inferred from Bayesian cluster analyses were used as input to MSVAR. It was important to test demographic changes at both these levels because departure from the Wright-Fisher assumption used in the MSVAR analytical model can create false signals [36]. We used only populations that contained at least 20 individuals, therefore restricting this analysis to the LGR (populations 1, 3, 4, 5, 15, 16, 17, 18, 21 and 25;
Additional files 2 and 3). Analyses at the gene pool level were performed only on gene pools presenting a low genetic substructure (see results) to avoid potential population structure bias [36]. We used very flexible priors in order to minimize their impact on the posterior [37]. All priors were specified from log Normal distributions as follows: $\mathrm{N}_{0}(4,6), \mathrm{N}_{1}(4,6), \mu(-4,3)$ and $\mathrm{T}_{\mathrm{a}}(3,6)$ (the first value represents the mean and the second value the standard deviation). Priors on the current and the ancestral population size were identical so that population decline or expansion is equally likely. An exponential model of population size change was assumed. The generation time was assumed to be 100 years (modal flowering age based on our field observations, data not shown). Three analyses with different starting points were carried out for each data set (1.2. $\times 10^{9}$ steps; thinning of 40000 steps). Convergence among MCMC chains was checked by applying the Gelam-Rubin statistic implemented in the CODA package in $R$ [38]. Samples from the three analyses were combined. The significance of changes in population size was examined using Bayes factors. The number of states in the chains in which the population has declined $\left(\mathrm{N}_{0} / \mathrm{N}_{1}<1\right)$ was divided by the number of states in which the population has expanded $\left(\mathrm{N}_{0} / \mathrm{N}_{1}>1\right)$ (see [37]). The means and the $95 \%$ highest probability densities (HPD) of the marginal posteriors of the natural parameters $\left(\mathrm{N}_{0}, \mathrm{~N}_{1}\right.$, $\mu$ and $\left.\mathrm{T}_{\mathrm{a}}\right)$ and the scaled parameters $\left(\theta_{0}=4 \mathrm{~N}_{0} \mu, \theta_{1}=4\right.$ $\mathrm{N}_{1} \mu$ and $\mathrm{t}_{\mathrm{f}}=\mathrm{Ta} / 2 \mathrm{~N}_{0}$ ) were obtained using Tracer v1.4.

\section{Results}

Bayesian clustering analyses - identification of major gene pools (sharp genetic discontinuities)

The likelihood of the data increased steadily from $K=1$ up to $K=5$ under the no-admixture model. A plateau was reached at $K=5$, above which the variance among replicates increased (Additional file 4). Only a few individuals remained unassigned to a gene pool for both $K=2$ (10 individuals, at a cut-off level of $\mathrm{P} \geq 0.8)$ and $K=3$, (12 individuals), while the number of unassigned individuals increased sharply for $K=4$ (102 individuals) and 5 (419 individuals) (Additional files 5 and 6). Hence, we considered that $K=3$ is the optimal solution for identifying major gene pools. These gene pools present characteristic geographic distributions (Figure 1 and Additional file 5). A first gene pool is distributed along the African littoral, but is absent from the Dahomey Gap and southern Central Africa regions. This distribution tracks the distribution of evergreen forests. A second gene pool extends from the drier zones of West Africa (including the Dahomey Gap) to the north-west of Cameroon. The third gene pool is restricted to the interior forests of Central Africa, reaching the littoral only in the south of Central Africa. It is noteworthy that the first 
two gene pools cluster together when two clusters are specified in the analysis $(K=2)$ (Additional file 5$)$.

All (but one) of the Central African E. ivorense samples (following [8]) and all West African samples that were originally assigned to E. ivorense (15 samples collected in the evergreen forest) were grouped within the first gene pool, which was denoted with the letter " $P$ " (Table 1). All West African samples a priori belonging to E. suaveolens (28 samples collected in the dry forest area) as well as samples from northwest Cameroon identified as E. suaveolens [8] were assigned to the second gene pool, which was therefore denoted " $S W$ " (Table 1). Finally, all central African samples identified as $E$. suaveolens, except individuals from northwest Cameroon included in $S W$, were assigned to the third gene pool, which was therefore denoted " $S C$ ".

Bayesian clustering analyses - identification of minor gene pools (diffuse genetic discontinuities)

Using the admixture model within each primary gene pool, the optimal $K$ reached $K=3$ within $I, K=1$ within $S W, K=2$ within $S C$. Hence, a total of six minor gene pools can be identified (Figure 1). They again formed geographically cohesive but more or less overlapping groups and a substantial number of individuals now appears admixed. According to their geographic distributions, the three minor gene pools detected within $I$ will be referred to as $I w$ (found in UGR and on the northwestern part of the LGR, Figure 1), In (found in the northern part of the LGR) and Is (found in the southern part of the LGR). The two minor gene pools detected

\begin{tabular}{|c|c|c|c|c|c|}
\hline \multirow{3}{*}{ Species } & \multirow{2}{*}{\multicolumn{4}{|c|}{ Major gene pools $(K=3)$}} & \multirow{3}{*}{ Total } \\
\hline & & & & & \\
\hline & SW & $S C$ & 1 & Unassigned & \\
\hline \multicolumn{6}{|l|}{ West Africa } \\
\hline E. ivorense & 0 & 0 & 15 & 0 & 15 \\
\hline E. suaveolens & 27 & 0 & 0 & 0 & 27 \\
\hline Unassigned & 1 & 0 & 9 & 0 & 10 \\
\hline Total & 28 & 0 & 24 & 0 & 52 \\
\hline \multicolumn{6}{|l|}{ Central Africa } \\
\hline E. ivorense & 0 & 1 & 96 & 0 & 97 \\
\hline E. suaveolens & 9 & 142 & 0 & 2 & 153 \\
\hline Unassigned & 3 & 252 & 81 & 10 & 346 \\
\hline Total & 12 & 395 & 177 & 12 & 596 \\
\hline
\end{tabular}

within $S C$ will be referred to as $S C n$ (northern LGR) and SCs (southern LGR) (Figure 1, Additional file 7). At a threshold of $80 \%$, approximately $40 \%$ of $I$ individuals could not be assigned to $I w$, In or Is, and approximately $20 \%$ of $S C$ individuals could not be assigned to $S C n$ or SCs (Additional file 7).

\section{Genetic diversity analyses}

Analyses used either the arbitrary-defined populations (local patterns of diversity) or the six minor gene pools (regional patterns of diversity) as the unit of classification. At the population level, significant departures from Hardy-Weinberg equilibrium were detected (excess of homozygotes). Mean null allele frequencies per population varied from 0.005 to 0.181 . Estimated selfing rate was close to zero in all but two populations. The two exceptions were populations 3 and 12, although these were not characterized by higher $F_{\text {IS }}$ (Additional file 2). Mean allelic richness varied from 1.33 to 10.70 .

At the gene pool level, the $F_{\mathrm{ST}}$ among populations differed significantly from zero in all but one gene pool (Is), but were always lower than 0.147 . Permutation tests on $R_{\mathrm{ST}}$ revealed no evidence of a phylogeographic signal in any of the gene pools except for Is (Table 2).

Overall, five of the 15 pairwise comparisons between the minor gene pools resulted in a significant phylogeographic signal $\left(R_{\mathrm{ST}}\right.$ significantly larger than $F_{\mathrm{ST}}$, Table 3$)$. These significant comparisons were generally population pairs from different major gene pools within the UGR and the LGR ( $I w-S C s, S W-S C n, S W-S C s)$. However a significant comparison was found within a major gene pool $(I w-I s)$, as well between two major gene pools in the LGR (Is-SCs).

\section{pDNA genetic diversity distribution and correspondence} with nuclear gene pools

Among the 297 sequences of the trnC-petN1R inter-genic fragment, 21 different haplotypes were identified (Figure 3, Additional file 8). A first clade of five related haplotypes (H1 to H5) was found along the coast of the LGR (H1), in Benin (H3 and H4), Ghana (H3), Guinea (H2 and H5), and the South of Ivory Coast (H5). H1, H2 and H5 were found only in I, with $\mathrm{H} 1$ in $I n$ and $I s$, and $\mathrm{H} 1, \mathrm{H} 2$ and $\mathrm{H} 5$ in $I w$, except one individual from southern Cameroon bearing $\mathrm{H} 1$ while unambiguously assigned to $\mathrm{SCn}$ (population 18, Additional files 2 and 3). $\mathrm{H} 3$ and $\mathrm{H} 4$ occurred in $S W$. The second clade includes 15 haplotypes (H6 to H21) which were found almost entirely in $S C$. There were two exceptions to this rule: $\mathrm{H} 15$ was found in a population from Ivory Coast (population 28, Additional files 2 and 3) from $S W$ gene pool; $\mathrm{H} 12, \mathrm{H} 20$ and $\mathrm{H} 21$ were found in northwest Cameroon (population 8, Additional files 2 and 3) from $S W$ gene pool too. Hence, within the major gene pool $I$, the parapatric minor gene pools $I n$ and $I s$ were 
Table 2 Population-level genetic diversity and differentiation at $\mathbf{n S S R s}$ within each minor gene pool

\begin{tabular}{|c|c|c|c|c|c|c|c|c|}
\hline Gene pool ${ }^{A}$ & N. populations & $N$ ind & $A_{\mathrm{O}}{ }^{\mathrm{B}}$ & $H_{\mathrm{O}}{ }^{\mathrm{C}}$ & $H_{\mathrm{S}}{ }^{\mathrm{D}}$ & $H_{\mathrm{T}}{ }^{\mathrm{E}}$ & $F_{\mathrm{ST}}{ }^{\mathrm{F}}$ & $R_{\mathrm{ST}}{ }^{\mathrm{G}}$ \\
\hline SCn & 9 & 305 & $3.24 \pm 1.08$ & 0.522 & 0.616 & 0.631 & $0.016^{*}$ & $0.002^{\mathrm{NS}}$ \\
\hline SCS & 6 & 95 & $3.26 \pm 1.02$ & 0.513 & 0.673 & 0.684 & $0.008^{N S}$ & $0.062^{*}$ \\
\hline SW & 5 & 40 & $N A^{\prime}$ & 0.542 & 0.598 & 0.651 & $0.099^{*}$ & $0.174^{\mathrm{NS}}$ \\
\hline In & 6 & 136 & $3.60 \pm 0.79$ & 0.423 & 0.726 & 0.749 & $0.043^{*}$ & $0.082^{\mathrm{NS}}$ \\
\hline Is ${ }^{H}$ & 3 & 46 & $N A^{\prime}$ & 0.435 & 0.586 & 0.605 & $0.056^{*}$ & $0.034^{\mathrm{NS}}$ \\
\hline$I^{H}$ & 3 & 20 & $3.49 \pm 1.04$ & 0.426 & 0.645 & 0.745 & $0.147^{*}$ & $-0.009^{\mathrm{NS}}$ \\
\hline
\end{tabular}

monomorphic for the same haplotype (H1) while the allopatric minor gene pool $I w$ also contained H1 East of the Dahomey gap (individuals located in Nigeria) and two other related haplotypes ( $\mathrm{H} 2$ and $\mathrm{H} 5)$ found only West of the Dahomey gap. Within the major gene pool $S C$, the parapatric $S C n$ and $S C s$ shared many haplotypes from the second clade, but with higher haplotype diversity in $S C s$ (12 haplotypes) than in $S C n$ (6 haplotypes), despite a larger sample size in the later. Gene pool $S W$ had mostly unique haplotypes from the first clade $(\mathrm{H} 3, \mathrm{H} 4)$ or the second clade (H12, H15) as well as haplotypes shared with gene pool $S C$ in their contact zone from North Cameroon (H20, H21).

Eight different haplotypes were characterized in the 72 sequences of the matK gene (Additional files 8 and 1). There was no clear genetic structuring over the Guinea region within $I$ (the haplotype $\mathrm{H} 4$ is represented in the UGR and the LGR) or over the LGR within SC. Five haplotypes were detected among the $66 \mathrm{trnL}$ sequences (Additional files 8 and 1). No polymorphism was found within $I$ or within $\mathrm{SCn}$. The geographic patterns of nucleo-cytoplasmic associations were consistent with those reported for the trnC-petN1R (Additional file 1). Levels of genetic polymorphism and differentiation were generally too low to allow estimation of divergence time between gene pools by molecular dating analyses, except between the $S C$ and $I$. Accession numbers corresponding to the $\operatorname{trn} C$, mat $K$ and $\operatorname{trn} L$ sequences are indicated in the Additional file 8.

Table 3 Pairwise genetic differentiation between minor gene pools estimated by $\boldsymbol{F}_{\mathrm{ST}}$ (lower diagonal matrix) and $\boldsymbol{R}_{\mathrm{ST}}$ (upper diagonal matrix)

\begin{tabular}{lllllll}
\hline & SW & SCn & SCs & Is & In & I $\boldsymbol{l}$ \\
\hline SW & & $0.342^{*}$ & $0.381^{*}$ & 0.146 & 0.149 & 0.197 \\
SCn & 0.223 & & 0.050 & 0.321 & 0.218 & 0.157 \\
SCs & 0.198 & 0.040 & & $0.286^{*}$ & 0.206 & $0.253^{*}$ \\
Is & 0.169 & 0.247 & 0.220 & & 0.117 & $0.296^{*}$ \\
In & 0.181 & 0.206 & 0.189 & 0.085 & & 0.100 \\
Iw & 0.190 & 0.215 & 0.160 & 0.100 & 0.080 & \\
\hline
\end{tabular}

${ }^{*}$ Test of $R_{\mathrm{ST}}>F_{\mathrm{ST}}$ statistically significant at $P<0.05$.

\section{Molecular dating analyses}

Posterior mean divergence times and 95\% posterior intervals are shown in Figure 2 and Table 4. The posterior mean age of the MRCA was $11.87 \mathrm{Ma}$ between $E$. ivorense (gene pool $I$ ) and Central African E. suaveolens (gene pool SC), 16.92 Ma between E. africanum and the common ancestor to E. ivorense and E. suaveolens, and 25.52 Ma for all Erythrophleum species.

\section{Demographic changes}

Evidence of demographic decline was detected by MSVAR analyses at both geographic scales (population and gene pool, Additional file 9). The data contained insufficient information to allow precise estimation of natural parameters $\mathrm{N}_{0}, \mathrm{~N}_{1}$, Ta and $\mu$. Marginal posterior distributions were wide and differed only slightly from prior distributions. By contrast, the scaled parameters $\theta_{1}$ and $t_{f}$ were well estimated with a narrow 95\% HPD (except for populations 17 and 21 and for SCs and In that presented wide 95\% HPDs), but not $\theta_{0}$ (except for populations 4, 16, 18 and for $\mathrm{SCn}$ ). A signal of population decline was detected in all populations and gene pools, mostly with strong support (Bayes Factor $>10$ in 10 cases, although $3<\mathrm{BF}<10$ in one case, and $0.33<\mathrm{BF}<3$, in three cases). Weak or no support corresponded to populations and gene pools in which the data were not very informative about scaled parameters.

\section{Discussion}

Major gene pools and species delimitation

In the present study, two major gene pools were expected, as samples came from two different species according to taxonomists. We unexpectedly detected three major gene pools, one corresponding to E. ivorense (gene pool $I$ ) and two corresponding to E. suaveolens (gene pools $S W$ and $S C$; Figure 1). Moreover, the order of appearance of the gene pools (with increasing values of $K$ ) was not consistent with the expected species delimitation because $I$ and $S W$ clustered together against $S C$ when we fixed $K=2$. This questions the monophyly of $E$. suaveolens and thus the botanical classification based on morphological characters. 


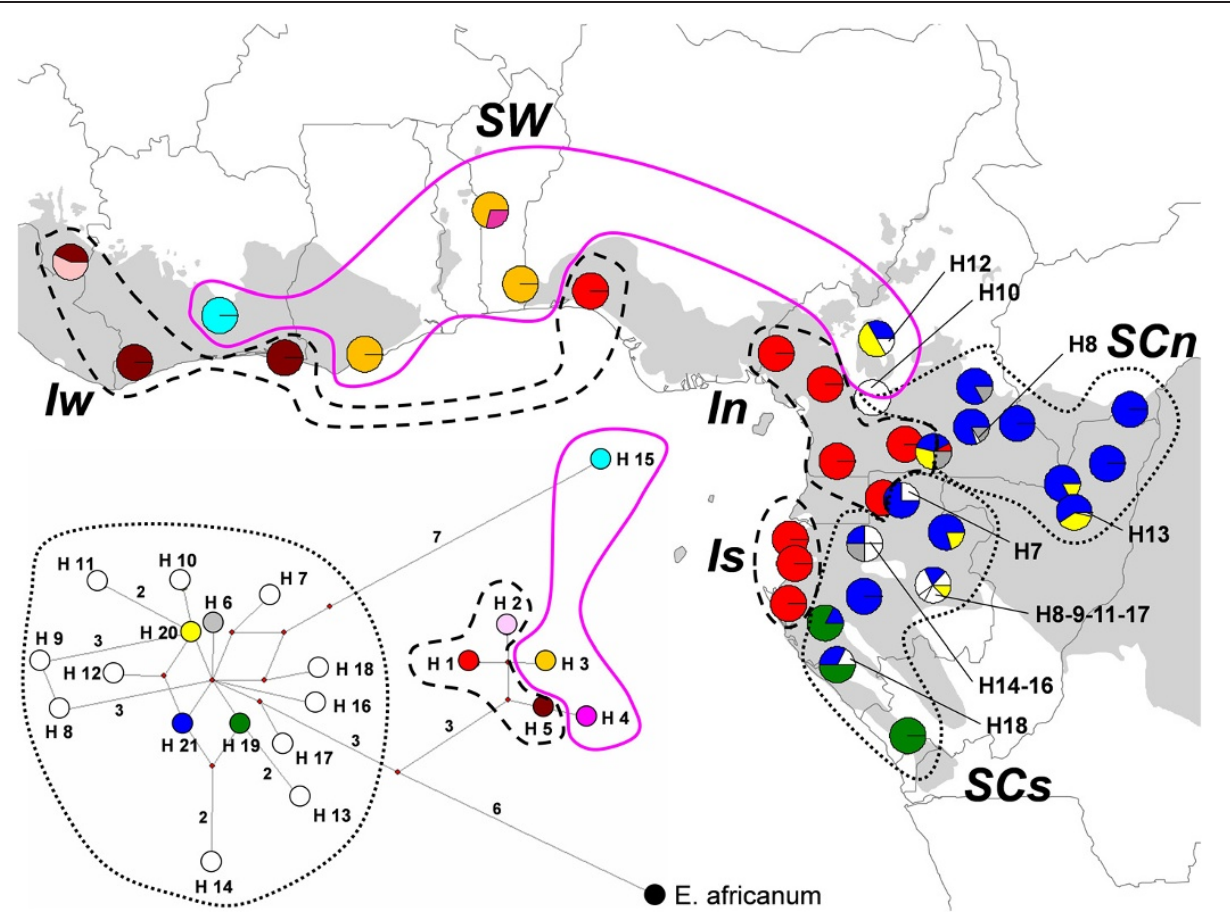

Figure 3 Distribution and median-joining network of haplotypes of the pDNA inter-genic fragment trnC-petN1R, and delimitation of the distribution of major and minor gene pools as inferred from nSSRs (pink line: SW gene pool, dashed line: SCn and SCs gene pools; dotted lines: I $\boldsymbol{w}, \mathbf{I n}$, Is gene pools). Rare haplotypes are indicated directly on the map by a number. Small dots on the haplotype network indicate ancestral nodes or unsampled haplotypes. Branch lengths are proportional to the number of mutations (numbers on branches where $>1$ mutation). Haplotypes associated with each of the three major gene pools are encircled on the haplotype network, except that haplotypes H12, H20 and H21, most typical of SC, are also found in SW populations from Northern Cameroon.

Gene pools $I$ and $S C$ present a long contact zone in Central Africa, so that the sharp genetic boundary indicates that they should correspond to distinct species following the BSC. The same can be argued between $I$ and $S W$ in West Africa. By contrast, $S W$ and $S C$ have a limited contact zone in northern Cameroon, but our limited sample size there does not allow us to confirm they are reproductively isolated. Additional sampling at their contact zone and a new taxonomic study based on morphological traits of E. suaveolens individuals from Central and West Africa would be justified to assess whether they might be considered as distinct morphological (sub-) species. Interestingly, significant phylogeographic signals (nSSRs, $R_{\mathrm{ST}}>F_{\mathrm{ST}}$ ) were mostly detected between major gene pools,

Table 4 Divergence time estimates of diversification events in Erythrophleum

\begin{tabular}{lll}
\hline & $\begin{array}{l}\text { Mean divergence } \\
\text { time }\end{array}$ & HPD95\% \\
\hline $\begin{array}{l}\text { African / non-African } \\
\text { Erythrophleum }\end{array}$ & 25.52 & {$[10.74-42.13]$} \\
$\begin{array}{l}\text { E. chlorostachys / E. fordii } \\
\text { E. africanum / (E. Ivorense - }\end{array}$ & 12.17 & {$[2.40-24.11]$} \\
E. suaveolens) & 16.92 & {$[4.36-31.39]$} \\
E. suaveolens / E. ivorense & 11.87 & {$[2.17-24.18]$} \\
\hline
\end{tabular}

suggesting ancient divergence (number of generations comparable or larger than the reciprocal of the mutation rate; [23]). This is in support of ongoing or accomplished speciation with a (near) complete barrier to gene flow.

The chloroplast genome was globally congruent with the nSSRs data in terms of haplotype distribution because each major gene pool generally carried private haplotypes. There are two exceptions however: in southern Cameroon, one individual from the $S C$ gene pool carried a haplotype typical of the $I$ gene pool, and in northern Cameroon, individuals belonging to the $S W$ gene pool had some of the haplotypes found in the $S C$ gene pool. These exceptions may result from past chloroplast captures or from incomplete lineage sorting. While incomplete lineage sorting can often impede reciprocal monophyly between fully isolated sister species with large population sizes, it is much less likely to maintain shared haplotypes and, moreover, it is unlikely to maintain a geographic correlation in the distribution of shared haplotypes between species. As all the reported exceptions are located close to contact zones between major gene pools, chloroplast capture appears a more parsimonious explanation. It suggests that individuals from the major gene pools might hybridize on rare occasion.

Examination of the distribution of pDNA lineages in each major gene pool reveals some additional incongruences 
because even the haplotypes unique to $S W$ are paraphyletic. Ancient chloroplast captures may also be responsible for this pattern, but incomplete lineage sorting is equally plausible. In any case, this supports rejection of the pDNA-based species delimitation based on the monophyly of pDNA lineages [8].

\section{Patterns of differentiation within the Erythrophleum genus in Africa: ecological gradient versus forest refuge hypothesis}

The three major gene pools are found in different habitats and climatic conditions. This is particularly true in West Africa where gene pool $I w$ was found in evergreen forests in locations where annual rainfall ranged from 1620 to $2350 \mathrm{~mm}$ (mean $=1980 \mathrm{~mm}$ ), while gene pool $S W$ was found in dry forests and in forest galleries with an annual rainfall ranging from 1055 to $1220 \mathrm{~mm}$ $($ mean $=1150 \mathrm{~mm})$. These results are fully consistent with the West African literature reporting that $E$. ivorense occurs only in wet evergreen forests while E. suaveolens occurs in much drier areas. In Central Africa, the contrast is less sharp: gene pools $I s$ and $I n$ were found in evergreen forests with very high annual rainfall (1620 to $3480 \mathrm{~mm}$; mean $=2460 \mathrm{~mm}$ ), while $S C$ occurred in the drier semievergreen forests (annual rainfall 1370 to $2050 \mathrm{~mm}$; mean $=1640 \mathrm{~mm}$ ), and $S W$ in the forest-savanna mosaic of northern Cameroon (annual rainfall 1530 to 2090 $\mathrm{mm}$; mean $=1710 \mathrm{~mm}$ ). Our data do not allow testing whether diversification has been driven by an ecological factor such as annual water availability, length of the dry season or another correlated ecological factor. However, given the above evidence and the fact that the third African species, E. africanum is only found in drier savanna habitats than E. suaveolens, we can conclude that there is a strong association between species diversification and habitat specialisation in African Erythrophleum, suggesting that the differentiation of major gene pools can be explained by the ecological gradient hypothesis.

Contrary to major gene pools, the diffuse genetic boundaries separating minor gene pools were not related to rainfall gradient, or any other obvious environmental gradient. There is thus no support for an ecological gradient hypothesis, in which the forms represent differentiated ecotypes which maintain some degree of gene flow.

Based on pDNA, Central African E. suaveolens (gene pool $S C$ ) and E. ivorense (I) diverged before the Pleistocene, during the Miocene or the Pliocene (Table 4). As, we examined a deep phylogeny, it seems reasonable to assume that sequence divergence times should closely approximate to species divergence times. Although posteriors are quite wide they support the view that genus diversification of African rainforest plants mostly preceded the Pleistocene glacial-interglacial cycles [39]. Evidence for the impact of Pleistocene climate changes may be present in intraspecific patterns of genetic diversity, but the polymorphism of the pDNA sequences was too low to date the divergence between major gene pools, and the polyphyletic nature of the chloroplast genome within gene pool $S W$ precludes any estimation of its divergence time.

\section{The origin of minor gene pools: population fragmentation and evidence of population decline support the forest refuge hypothesis}

Molecular dating did not allow estimation of divergence times between minor gene pools. However, they should be much inferior to the divergence time between major gene pools, and are thus likely to be contemporaneous of the Pleistocene climatic cycles. The $R_{\mathrm{ST}}$ values provide insights on the relative divergence. Indeed, $I w$ presents a significant phylogeographic signal with respect to Is. Differentiation should thus be much more ancient than the differentiation between $I n$ and $I s$, or between $S C n$ and $S C$, where minor gene pools are in direct contact and there is a high degree of admixture.

Additional insights come from tests of demographic changes. Indeed, we detected a signal of population decline (nSSR data) in all the LGR gene pools, both at the population and gene pool levels, which confirms previous results obtained with pDNA data [8]. This signal is consistent with the forest refuge hypothesis. However, as it could not be accurately dated, we cannot state with certainty that this was driven by climate changes.

Several studies of tree species have reported patterns of genetic differentiation between the northern and the southern part of the LGR with a disjunction located approximately between $0^{\circ} \mathrm{N}$ and $2^{\circ} \mathrm{N}$, despite the current absence of a habitat discontinuity [5-7,40]. We also found this pattern of differentiation with a limit close to $2^{\circ} \mathrm{N}$, for both Erythrophleum species. Although different factors can generate similar patterns, this convergence among several forest tree species suggests ancient habitat fragmentation, consistent with the forest refuge hypothesis, if we assume the existence of northern and southern refuges. The weak genetic differentiation between the northern and southern gene pools, the absence of phylogeographic signal, and the large proportion of admixed individuals near the contact zone indicate that the LGR minor gene pools within each species are only weakly differentiated and have already partly introgressed.

\section{The relevance of the Dahomey Gap for the differentiation of rainforest species}

The Dahomey Gap is often considered as the limit between Upper and Lower Guinean phytogeographic regions. However, it does not coincide with the delimitation of $S W$ and Iw gene pools (Figures 1 and 3). This result is not surprising for E. suaveolens given that, in West Africa, it mainly occurs in gallery forests within dry forest or savanna 
landscapes at the periphery of the Guineo-Congolian forest. However, for $E$. ivorense, which is strictly associated with evergreen forests with high rainfall, the distribution of $I w$ on each part of the Dahomey gap is more surprising. In fact, for the nuclear genome, a genetic discontinuity occurs West of the Dahomey gap, in Nigeria (limit between gene pools $I w$ and $I n)$, while it occurs at the height of the Dahomey gap for the plastid genome (distribution limit of haplotypes $\mathrm{H} 1$ and H5; Figure 3). This pattern supports the hypothesis that populations on each part of the Dahomey Gap were recently in contact and that the savanna corridor reopened recently. Meanwhile, the absence of shared pDNA haplotypes between the LGR I $w$ and the UGR I $w$ suggests that the Dahomey gap did play a role in the past. It has been suggested that the Dahomey Gap was dominated by savanna during the glacial periods and by rainforest during the last interglacial and the early Holocene $[12,14]$. The current savanna corridor was almost certainly present ca. 1100 years ago [14] but in southern Benin a semi-evergreen rainforest prevailed between $c a .8400$ and 4500 years BP [14]. Thus, the distribution of $I w$ on each part of the Dahomey gap and the absence of shared pDNA haplotypes between UGR and LGR populations indicate that the populations were separated in the past during a cooling event. They underwent secondary contact during a warming event and separated again during the last opening of the Dahomey Gap.

The discrepancy between the positions of the nuclear and plastid genetic discontinuities could be explained by more limited seed than pollen dispersal and a phenomenon of chloroplast capture when populations west of the Dahomey gap $(I w)$ extended to the east during the more humid periods and hybridized with eastern populations bearing the $\mathrm{H} 1$ haplotype. Additional sampling in Nigeria would be necessary to test this hypothesis. Interestingly, the limit between $I w$ and $I n$ is localized somewhere between $5^{\circ}$ and $8^{\circ} \mathrm{E}$ (Nigeria), a biogeographic limit coinciding with the one found in lowland forest bird taxa [41].

\section{Conclusion}

Our study provides new insights into patterns of differentiation that occurred within evergreen, semi-evergreen and forest galleries of the Guinea region using a widespread tree genus. We detected three major gene pools occurring in distinct habitat types that probably originated before the Pleistocene. Hence, climatic gradients in tropical Africa are probably the first major driver of differentiation in the genus Erythrophleum, but additional tests based on molecular signatures of climate-driven selection would be necessary to confirm this hypothesis. Furthermore, within major gene pools, we provide evidence of historical barriers to gene flow (i) within the evergreen and semi-evergreen forests of the LGR at a latitude between 0 and $2^{\circ} \mathrm{N}$, (ii) within the evergreen forest of southern Nigeria at a longitude between 5 and $8^{\circ} \mathrm{E}$. The first barrier has been reported in other African rainforest tree species and could indicate the occurrence of Northern and Southern forest refuges in the LGR. The forest refuge hypothesis is also supported by genetic signatures of past population declines. Contrary to a priori expectations, the second barrier does not coincide with the Dahomey gap, but to the east of this region, indicating that this savanna corridor was previously dominated by an evergreen forest containing E. ivorense. Nevertheless, the occurrence of an east-west genetic discontinuity indicates that West African and Central African forests have probably been isolated most of the time during the Pleistocene. Finally, while three gene pools with sharp genetic boundaries have been detected, chloroplast captures seem to have occurred in a few instances at their contact zones, suggesting that they may not be fully isolated.

\section{Data accessibility}

DNA sequences: accessions numbers provided in Additional file 8.

\section{Additional files}

Additional file 1: Spatial distribution of the genetic diversity observed for matK (above) and for trnL (below). Both figures show the median joining network for $E$. suaveolens and E. ivorense rooted on E. africanum, E. chlorostachys and E. fordii.

Additional file 2: Sampling locations, diversity data, null allele frequencies and selfing rate estimates.

Additional file 3: Delimitation of populations. This figure represents the spatial distribution of the individuals included in this study and their clustering in populations.

Additional file 4: Likelihood increasing mean according to $\mathrm{K}$ and DeltaK Evanno's method. These figures represent likelihood increasing mean according to $\mathrm{K}$ and DeltaK.

Additional file 5: Identification of major gene pools: spatial distribution of the individuals assigned to each gene pool (at a threshold probability of $\mathbf{8 0} \%$ ) with increasing values of $\mathrm{K}$. These figures represent the probability assignments of each individuals with increasing values of $\mathrm{K}$ (from 2 to 5), and the distribution of each cluster on the map of the region.

Additional file 6: Assignment of the individuals to gene pools at a probability threshold of $\mathbf{8 0 \%}$ with increasing values of $\mathbf{K}$. The table provides numerical values of data represented in Additional file 5.

Additional file 7: Map of admixture zone between the Central African Northern and Southern minor gene pools in E. ivorense and E. suaveolens.

Additional file 8: GenBank accessions numbers of the plastid DNA sequences obtained in this study. The table provides the accession numbers for all pDNA haplotypes described in this study.

Additional file 9: Demographic inferences obtained by MSVAR analyses at a population and gene-pool level.

\section{Competing interests}

The authors declare that they have no competing interests. 


\section{Authors' contributions}

The research interests of JD, E-EBKE, J-LD and OJH are primarily in studying the evolution of tree species from tropical African rainforests. RPB is primarily interested in statistical aspects of evolutionary genetics and in examining how major physical events have influenced speciation and local patterns of diversity, particularly in reptiles. PM has a general interest in the analysis of DNA sequence variation for studying evolution, with current research focused mainly on comparing multi-locus phylogeographic patterns among different temperate and cold-adapted herbivorous insects. All authors read and approved the final manuscript.

\section{Acknowledgments}

Research funded by the Gembloux Agricultural University (FUSAGx, Belgium via project PPR 10.000, the FNRS (grants FRFC 2.4.576.07., 2.4577.10 and MIS F.4.519.10.F) and by the C3A project financed by the French ANR (Agence Nationale de la Recherche) under the ANR-BIODIV program. For their help with sampling, we also thank Guillaume Koffi, Lambert Kouadio, Charles Doumenge, Myriam Heuertz, Michel Arbonnier, Patrice Koumba Ipandi, Jean François Gillet, Nils Bourland, Kasso Dainou, Gilles Dauby, 'équipe Nature + Pallisco', Gilbert Todou, Raymonde Mboma. We also thank the logging companies: Pallisco, Wijma, Rougier, Precious Woods for allowing access to their concessions and ENEF (Ecole Nationale des Eaux et Forêts) for facilitating sampling in Gabon. Thanks to Garry Cook for samples of $E$. chlorostachys from Australia and Chonglu Zhong and Zhigang Zhao for samples of $E$. fordii from China.

\section{Author details}

${ }^{1}$ Service Evolution Biologique et Ecologie, CP160/12, Faculté des Sciences, Université Libre de Bruxelles, 50 Av. F. Roosevelt, 1050 Brussels, Belgium. ${ }^{2}$ Bioversity International, Forest Genetic Resources Programme, Sub-Regional Office for Central Africa, P.O. Box 2008 Messa, Yaoundé, Cameroon. ${ }^{3}$ School of Natural Sciences and Psychology, Liverpool John Moores University, Liverpool L3 3AF, UK. ${ }^{4}$ Faculté des Sciences et Techniques, BP 4521, Université d'Abomey-Calavi, Abomey-Calavi, Benin. ${ }^{5}$ Laboratoire de Foresterie des Régions Tropicales et Subtropicales, Unité de Gestion des Ressources Forestières et des Milieux Naturels, Gembloux Agro-Bio Tech, Université de Liège, Passage des Déportés 5030 Gembloux, Belgium.

Received: 22 May 2013 Accepted: 20 August 2013

Published: 12 September 2013

\section{References}

1. White F: The vegetation of Africa: A Descriptive Memoir to Accompany the UNESCO/AETFAT/UNSO Vegetation Map of Africa, Natural Resources Research no. 20. Paris: UNESCO; 1983

2. White F: The Guineo-Congolian region and its relationships to other phytochoria. Bulletin du jardin botanique nationale de Belgique 1979, 49:11-55.

3. Gomez C, Dussert S, Hamon P, Hamon S, De Kochko A, Poncet V: Current genetic differentiation of Coffea canephora Pierre ex A. Froehn in the Guineo-Congolian African zone: cumulative impact of ancient climatic changes and recent human activities. BMC Evol Biol 2009, 9:167.

4. Born C, Alvarez N, McKey D, Ossari S, Wickings EJ, Hossaert-McKey M, Chevallier $\mathrm{MH}$ : Insights into the biogeographical history of the lower Guinea forest domain: evidence for the role of refugia in the intraspecific differentiation of Aucoumea klaineana. Mol Ecol 2010, 20:131-142.

5. Dainou K, Bizoux JP, Doucet JL, Mahy G, Hardy OJ, Heuertz M: Forest refugia revisited: $n S S R s$ and $c p D N A$ sequences support historical isolation in a wide-spread African tree with high colonization capacity, Milicia excelsa (Moraceae). Mol Ecol 2010, 19:4462-4477.

6. Dauby G, Duminil J, Heuertz M, Hardy OJ: Chloroplast DNA polymorphism and phylogeography of a Central African tree species widespread in mature rainforests: Greenwayodendron suaveolens (Annonaceae). Trop Plant Biol 2010, 3:4-13.

7. Debout GDG, Doucet JL, Hardy OJ: Population history and gene dispersal inferred from spatial genetic structure of a Central African timber tree, Distemonanthus benthamianus (Caesalpinioideae). Heredity 2010, 104.

8. Duminil J, Heuertz M, Doucet JL, Bourland N, Cruaud C, Gavory F, Doumenge C, Navascues M, Hardy OJ: CpDNA-based species identification and phylogeography: application to African tropical tree species. Mol Ecol 2010, 19:5469-5483.

9. Freedman AH, Thomassen HA, Buermann W, Smith TB: Genomic signals of diversification along ecological gradients in a tropical lizard. Mol Ecol 2010, 19:3773-3788.

10. Holstein N, Renner SS: A dated phylogeny and collection records reveal repeated biome shifts in the African genus Coccinia (Cucurbitaceae). BMC Evol Biol 2011, 11:28.

11. Marret F, Scourse J, Jansen JHF, Schneider R: Climate and palaeoceanographic changes in west Central Africa during the last deglaciation: palynological investigation. Cr Acad Sci ll A 1999, 329:721-726.

12. Dupont LM, Bonner B, Schneider R, Wefer G: Mid-Pleistocene environmental change in tropical Africa began as early as $1.05 \mathrm{Ma}$. Geology 2001, 29:195-198.

13. Demenocal P, Ortiz J, Guilderson T, Adkins J, Sarnthein M, Baker L, Yarusinsky M: Abrupt onset and termination of the African humid period: rapid climate responses to gradual insolation forcing. Quaternary Sci Rev 2000, 19:347-361.

14. Salzmann U, Hoelzmann P: The dahomey Gap: an abrupt climatically induced rain forest fragmentation in West Africa during the late holocene. Holocene 2005, 15:190-199.

15. Vincens $A$, Buchet $G$, Servant M: Vegetation response to the "african Humid Period" termination in Central Cameroon $\left(7^{\circ} \mathrm{N}\right)$ - new pollen insight from Lake Mbalang. Clim Past 2010, 6:281-294.

16. Akoègninou A, Van Der Burg WJ, Van Der Maesen LJG (Eds): Flore Analytique Du Bénin. Leiden, Netherlands: Backhuys Publishers; 2006:1034

17. Aubréville A: La flore forestière de la Côte d'Ivoire, Tome 1. Nogent-surMarne, Centre technique forestier tropical (Ctft) 1959, 1:370

18. Aubréville A: Légumineuses-Caesalpinioidées, in Aubréville A. \& Leroy J.-F., Flore du Cameroun 9. Paris: Muséum national d'Histoire naturelle; 1970:1-339.

19. Hawthorne WD, Jongkind C: Guide to the Woody Plants of Western Africa. Kew, U.K.: Royal Botanic Gardens; 2006.

20. Duminil J, Koffi KG, Debout GDG, Sebastiani F, Vendramin GG, Heuertz M, Gonzalez-Martinez SC, Hardy OJ: Isolation of SSR markers for two African tropical tree species Erythrophleum suaveolens and E. ivorense (Caesalpinioideae). Am J Bot Prim Notes Pro 2011, 98:E106-E108.

21. Goudet J: FSTAT (Version 1.2): a computer program to calculate F-statistics. J Hered 1995, 86:485-486.

22. Dempster AP, Laird NM, Rubin DB: Maximum likelihood from incomplete data via the EM algorithm. J R Stat Soc 1977, 39:1-38.

23. Chapuis M-P, Estoup A: Microsatellite null alleles and estimation of population differentiation. Mol Biol Evol 2007, 24:621-631.

24. Pritchard JK, Stephens M, Donnelly P: Inference of population structure using multilocus genotype data. Genetics 2000, 155:945-959.

25. Falush D, Stephens M, Pritchard JK: Inference of population structure using multilocus genotype data: linked loci and correlated allele frequencies. Genetics 2003, 164:1567-1587.

26. Jakobsson M, Rosenberg NA: CLUMPP: a cluster matching and permutation program for dealing with label switching and multimodality in analysis of population structure. Bioinformatics 2007, 23:1801-1806

27. Evanno G, Regnaut S, Goudet J: Detecting the number of clusters of individuals using the software structure: a simulation study. $\mathrm{Mol}$ Ecol 2005, 14:2611-2620.

28. Hardy OJ, Vekemans X: SPAGEDi: a versatile computer program to analyse spatial genetic structure at the individual or population levels. Mol Ecol Notes 2002, 2:618-620

29. Hardy OJ, Charbonnel N, Freville H, Heuertz M: Microsatellite allele sizes: a simple test to assess their significance on genetic differentiation. Genetics 2003, 163:1467-1482.

30. Taberlet P, Gielly L, Pautou G, Bouvet J: Universal primers for amplification of three non-coding regions of chloroplast DNA. Plant Mol Biol 1991, 17:1105-1109.

31. Bandelt $\mathrm{H}-\mathrm{J}$, Forster $\mathrm{P}$, Röhl A: Median-joining networks for inferring intraspecific phylogenies. Mol Biol Evol 1999, 16:37-487.

32. Bruneau A, Mercure M, Lewis GP, Herendeen PS: Phylogenetic patterns and diversification in the caesalpinioid legumes. Can J Bot 2008, 86:697-718.

33. Drummond AJ, Rambaut A: BEAST: Bayesian evolutionary analysis by sampling trees. Bmc Evol Biol 2007, 7:214.

34. Posada, J. (2008). jModelTest: Phylogenetic Model Averaging. Molecular Biology \& Evolution, 25(7), 1253-1256. 
35. Storz JF, Beaumont MA: Testing for genetic evidence of population expansion and contraction: an empirical analysis of microsatellite DNA variation using a hierarchical Bayesian model. Evolution 2002, 56:154-166.

36. Chikhi L, Sousa VC, Luisi P, Goossens B, Beaumont MA: The confounding effects of population structure, genetic diversity and the sampling scheme on the detection and quantification of population size changes. Genetics 2010, 186:983-995.

37. Girod C, Vitalis R, Leblois R, Freville H: Inferring population decline and expansion from microsatellite data: a simulation-based evaluation of the MSVAR method. Genetics 2011, 188:165-U287.

38. Ihaka A, Gentleman R: R: a language for data analysis and graphics. J Comput Graph Stat 1996, 5:299-314.

39. Plana V: Mechanisms and tempo of evolution in the African GuineoCongolian rainforest. Philos T Roy Soc B 2004, 359:1585-1594.

40. Ndiade Bourobou D: Dynamique Spatiale et Temporelle de la Diversité Génétique d'une espèce rare en Afrique Centrale: Baillonella toxisperma Pierre (le Moabi). France: PhD, Montpellier University; 2011.

41. Mayr E, O'Hara RJ: The biogeographic evidence supporting the Pleistocene forest refuge hypothesis. Evolution 1986, 40:55-67.

doi:10.1186/1471-2148-13-195

Cite this article as: Duminil et al:: Large-scale pattern of genetic differentiation within African rainforest trees: insights on the roles of ecological gradients and past climate changes on the evolution of Erythrophleum spp (Fabaceae). BMC Evolutionary Biology 2013 13:195.

\section{Submit your next manuscript to BioMed Central and take full advantage of:}

- Convenient online submission

- Thorough peer review

- No space constraints or color figure charges

- Immediate publication on acceptance

- Inclusion in PubMed, CAS, Scopus and Google Scholar

- Research which is freely available for redistribution 